\title{
Research Article \\ Stability Guaranteed Active Fault-Tolerant Control of Networked Control Systems
}

\author{
Shanbin Li, Dominique Sauter, Christophe Aubrun, and Joseph Yamé \\ Centre de Recherche en Automatique de Nancy (CRAN-UMR 7039), Nancy Université, CNRS, BP 239, \\ 54506 Vandoeuvre-lès-Nancy Cedex, France \\ Correspondence should be addressed to Joseph Yamé, joseph.yame@cran.uhp-nancy.fr
}

Received 4 May 2007; Revised 15 October 2007; Accepted 9 December 2007

Recommended by Jakob Stoustrup

The stability guaranteed active fault-tolerant control against actuators failures and plant uncertainties in networked control systems (NCSs) is addressed. A detailed design procedure is formulated as a convex optimization problem which can be efficiently solved by existing software. An illustrative example is given to show the efficiency of the proposed method for network-based control for uncertain systems.

Copyright (c) 2008 Shanbin Li et al. This is an open access article distributed under the Creative Commons Attribution License, which permits unrestricted use, distribution, and reproduction in any medium, provided the original work is properly cited.

\section{INTRODUCTION}

Fault-tolerant control (FTC) techniques against actuator faults can be classified into two groups [1]: passive and active approaches. In passive FTC systems, a single controller with fixed structure/parameters is used to deal with all possible failure scenarios which are assumed to be known a priori. Consequently, the passive controller is usually conservative. Furthermore, if a failure out of those considered in the design occurs, the stability and performance of the closed-loop system might not be guaranteed. Such potential limitations of passive approaches provide a strong motivation for the development of methods and strategies for active FTC (AFTC) systems.

In contrast to passive FTC systems, AFTC techniques rely on a real-time fault detection and isolation (FDI) scheme and a controller reconfiguration mechanism. Such techniques allow a flexibility to select different controllers according to different component failures, and therefore better performance of the closed-loop system can be expected. However, this holds true when the FDI process does not make an incorrect or delayed decision [2]. Some preliminary results have been obtained on AFTC which is immune to imperfect FDI process $[3,4]$. In [5], the latter issue is further discussed in a classical setting (i.e., point-to-point control) by using the guaranteed cost control approach and online controller switching in or- der to ensure stability of the closed-loop system at all times. The aim of this paper is to extend the results in [5] to uncertain plants controlled over digital communication networks. In such networks, the information transfer from sensors to controllers and from controllers to actuators is not instantaneous but suffers communication delays. Such communication delays can be highly variable due to their strong dependence on variable network conditions such as congestion and channel quality. These network-induced delays may impact adversely on the stability and performance of the control system $[6,8]$. Networked control systems (NCSs) are now pervasive (see, e.g., the recent special issue of the proceedings of the IEEE [7]), and such systems are long-running real-time systems which should function in a correct manner even in the presence of failures. This makes the issue of fault tolerant control in NCS an important one and entails designing strategies to cope with some of the fundamental problems introduced by the network such as bandwidth limitations, quantization and sampling effects, message scheduling and communication delays. Motivated by the above considerations, we address the problem of fault tolerant control in NCSs with time-varying delays. Specifically, we extend the results of [5] for the stabilization of a plant, subject to model uncertainties and actuator faults, which is controlled over a communication network that induces time-varying but bounded delays. 


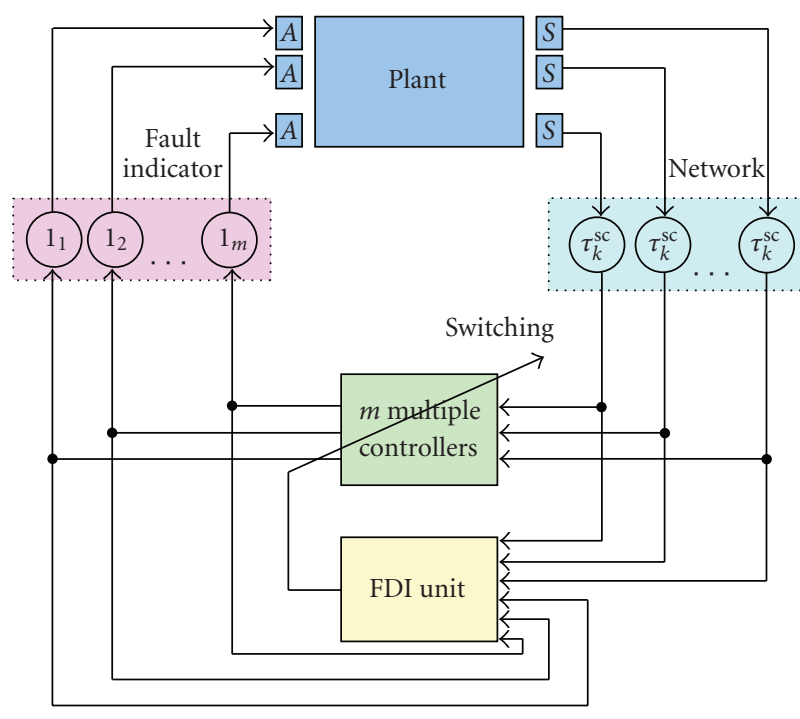

FIGURE 1: Networked control system with actuator failures.

The outline of the paper is as follows. In Section 2, a network-based control model for an uncertain plant subject to actuator failures is proposed and the guaranteed cost control problem is formulated. In Section 3, the detailed procedure for designing the NCSs-based fault-tolerant controller is given. Section 4 presents a design example to illustrate the benefit of the proposed FTC design procedure. Finally, conclusions are given in Section 5. The proof of the main theorem of the paper is reported in the appendix.

\section{PROBLEM STATEMENT}

Figure 1 shows the basic networked control architecture which is considered in this paper and which consists of a single uncertain plant, with few sensors and actuators, controlled by a digital controller in a centralized structure.

The delays induced by the network in the closedloop control system are modeled as time-varying quantities $\tau(k)=\tau_{k}^{\mathrm{sc}}$ arising from the communication delays between sensors and controllers at time $k$. Without loss of generality, we assume that there are no transmission time-delays between the controllers and the actuators. The actuators might be subject to faults during the system operation. Thus, taking into account the potential failures of actuators, the interconnection of the uncertain discrete-time plant and a discretetime controller through the digital communication link as depicted in Figure 1 can be described by the following dynamical and state-delayed feedback equations:

$$
\begin{aligned}
x(k+1) & =(A+D \Delta(k) E) x(k)+B \mathcal{L} u(k), \\
x(0) & =x_{0}, \\
u(k) & =K x(k-\tau(k)),
\end{aligned}
$$

where $x(k) \in \mathcal{R}^{n}$ is the state of the uncertain plant, $u(k) \in$ $\mathcal{R}^{m}$ is the control input, $A, B, D, E$ are all real constant matrices, and matrix $K$ is the controller gain matrix to be designed. The time-varying matrix $\Delta(k)$ represents norm- bounded parameter uncertainties and satisfies the bound $\Delta(k)^{T} \Delta(k) \leq I$ where $I$ denotes the identity matrix with appropriate dimension. The constant matrices $D$ and $E$ characterize the structure of these parameter uncertainties. The fault indicator matrix $\mathcal{L}$ is given by

$$
\mathscr{L}=\operatorname{diag}\left\{l_{1}, \ldots, l_{m}\right\}
$$

with $l_{j} \in\{0,1\}$ for $j=1,2, \ldots, m$, where $l_{j}=1$ means that the $j$ th actuator is in healthy state, whereas the $j$ th actuator is meant to experience a total failure when $l_{j}=0$. Having a finite number of actuators, the set of possible related failure modes is also finite and, by abuse of notation, we denote this set by

$$
\mathscr{L}=\left\{\mathscr{L}^{1}, \mathcal{L}^{2}, \ldots \mathscr{L}^{N}\right\}
$$

with $N=2^{m}-1$. Each failure mode $\mathcal{L}^{i},(i=1,2 \ldots, N)$ is therefore an element of the set $\mathcal{L}$. We also view $\mathcal{L}^{i}$ as a matrix, that is, as a particular pattern of matrix $\mathcal{L}$ in (4) depending on the values of $l_{j}(j=1,2, \ldots m)$. Throughout, when $\mathcal{L}$ is invoked as a matrix, it will mean that matrix $\mathcal{L}$ varies over the set of matrices in (5). Note that the faulty mode $\mathcal{L}^{i}$ in the NCS architecture of Figure 1 is estimated by the FDI unit. In order to ensure that system (1) should remain controllable, we assume that the set $\mathcal{L}$ excludes the element $\operatorname{diag}\{0,0, \ldots, 0\}$, that is, at least one actuator should be healthy.

We further assume that the time-varying delays $\tau(k)$ lie between the following positive integer bounds $\tau_{m}$ and $\tau_{M}$, that is,

$$
\tau_{m} \leq \tau(k) \leq \tau_{M} .
$$

Given positive definite symmetric matrices $Q_{1}$ and $Q_{2}$, we consider the quadratic cost function

$$
J=\sum_{k=0}^{\infty}\left[x^{T}(k) Q_{1} x(k)+u^{T}(k) Q_{2} u(k)\right]
$$

and with respect to this cost function, we define the guaranteed cost controller in the event of actuator failures as follows.

Definition 1. If there exists a control law $u(k)$ and a positive scalar $J^{*}$ such that for all admissible uncertainties $\Delta(k)$ and all failure modes $\mathcal{L}^{i} \in \mathcal{L}$ the closed-loop system (1)-(3) is stable with cost function (7) satisfying $J \leq J^{*}$, then $J^{*}$ is said to be a guaranteed cost and $u(k)$ a guaranteed cost controller for the uncertain system (1).

In Section 3, we will proceed through two main steps to design a cost guaranteed active fault tolerant control in the NCS framework. These steps are

(i) construct a fault-tolerant controller (i.e., a robust controller), with structure as given by (3), which achieves the smallest possible value for $J^{*}$ under all admissible plant uncertainties and all actuator failure modes;

(ii) redesign that part of the above controller associated to only one fault-free actuator in order to improve the robust performance without loss of the stability property of the design in step (i); step (ii) repeats for all $m$ actuators and results in a bank of $m$ controllers. 
It follows from inequality $m \leq N=2^{m}-1$, that the cardinality of the bank of controllers (which is equal to the number of actuators) is less than the cardinality of the set $\mathcal{L}$ of faulty modes. For each faulty mode $\mathcal{L}^{i}$, the controller to be switched on should be the best as ranked with respect to a closed-loop performance index. In this paper, we will not address the switching and reconfiguration mechanisms; we focus on the design of the bank of $m$ controllers.

\section{AFTC DESIGN FOR NCS}

\subsection{Robust stability}

In this subsection, we establish a sufficient condition for the existence of a guaranteed cost network-based controller for the uncertain plant (1). Note that the control law (3) applied to plant (1) results in the following system:

$$
x(k+1)=A_{1} x(k)+B \mathcal{L} K x(k-\tau(k)),
$$

where $A_{1}=A+D \Delta(k) E$. The cost function associated to system (8) is therefore

$$
J=\sum_{k=0}^{\infty} x_{e}^{T}(k) Q x_{e}(k)
$$

where $x_{e}^{T}(k)=\left[x^{T}(k), x^{T}(k-\tau(k))\right]$ and $Q=\operatorname{diag}\left\{Q_{1}, K^{T} Q_{2} K\right\}$. Under the assumptions made in Section 2, we can state the following result.

Theorem 1. If there exist a gain matrix $K$, a scalar $\epsilon>0$, symmetric positive-definite matrices $P_{1} \in \mathcal{R}^{n \times n}, R \in \mathcal{R}^{n \times n}, S \in$ $\mathcal{R}^{n \times n}$, and matrices $P_{2} \in \mathcal{R}^{n \times n}, P_{3} \in \mathcal{R}^{n \times n}, W \in \mathcal{R}^{2 n \times 2 n}$, $M \in \mathcal{R}^{2 n \times n}$ such that the following matrix inequalities are satisfied:

$$
\begin{gathered}
{\left[\begin{array}{ccc}
\Gamma & P^{T}\left[\begin{array}{c}
0 \\
B \mathcal{L} K
\end{array}\right]-M & {\left[\begin{array}{c}
E^{T} \\
0
\end{array}\right]} \\
* & -R+K^{T} Q_{2} K & 0 \\
* & * & -\epsilon I
\end{array}\right]<0,} \\
{\left[\begin{array}{cc}
W & M \\
* & S
\end{array}\right] \geq 0}
\end{gathered}
$$

with

$$
\begin{aligned}
\Gamma= & P^{T}\left[\begin{array}{cc}
0 & I \\
A-I & -I
\end{array}\right]+\left[\begin{array}{cc}
0 & I \\
A-I & -I
\end{array}\right]^{T} P \\
& +\epsilon P^{T}\left[\begin{array}{cc}
0 & 0 \\
0 & D D^{T}
\end{array}\right] P+\left[\begin{array}{cc}
\mu R+Q_{1} & 0 \\
0 & P_{1}+\tau_{M} S
\end{array}\right] \\
& +\tau_{M} W+[M \quad 0]+\left[\begin{array}{cc}
M & 0
\end{array}\right]^{T}, \\
\mu= & +\left(\tau_{M}-\tau_{m}\right), \quad P=\left[\begin{array}{cc}
P_{1} & 0 \\
P_{2} & P_{3}
\end{array}\right],
\end{aligned}
$$

then system (8) is asymptotically stable and the cost function (9) satisfies the inequality

$$
\begin{aligned}
J \leq & x^{T}(0) P_{1} x(0)+\sum_{l=-\tau_{M}}^{-1} x^{T}(l) R x(l) \\
& +\sum_{\theta=-\tau_{M}+1}^{0} \sum_{l=-1+\theta}^{-1} y^{T}(l) S y(l) \\
& +\sum_{\theta=-\tau_{M}+2}^{-\tau_{M}+1} \sum_{l=\theta-1}^{-1} x^{T}(l) R x(l)
\end{aligned}
$$

where $y(l)=x(l+1)-x(l)$.

Proof. See the appendix.

Remark 2. The “*” sign represents blocks that are readily inferred by symmetry.

Remark 3. Note that the upper bound in (13) depends on the initial condition of system (8). To remove the dependence on the initial condition, we suppose that the initial state of system (8) might be arbitrary but belongs to the set $\delta=\{x(l) \in$ $\left.\mathcal{R}^{n}: \quad x(l)=U v, v^{T} v \leq 1, l=-\tau_{M},-\tau_{M}+1, \ldots,-\tau_{m}\right\}$, where $U$ is a given matrix. Inequality (13) leads to

$$
J \leq \lambda_{\max }\left(U^{T} P_{1} U\right)+\rho_{1} \lambda_{\max }\left(U^{T} R U\right)+\rho_{2} \lambda_{\max }\left(U^{T} S U\right),
$$

where $\lambda_{\max }(\cdot)$ denotes the maximum eigenvalue of matrix $(\cdot), \rho_{1}=\mu\left(\tau_{M}+\tau_{m}\right) / 2$, and $\rho_{2}=2 \tau_{M}\left(\tau_{M}+1\right)$.

\subsection{Step (i): Controller Design}

Now, we derive the guaranteed cost controller in terms of the feasible solutions to a set of linear matrix inequalities.

Using Sherman-Morrison matrix inversion formula, we have

$$
P^{-1}=\left[\begin{array}{cc}
P_{1}^{-1} & 0 \\
-P_{3}^{-1} P_{2} P_{1}^{-1} & P_{3}^{-1}
\end{array}\right] .
$$

In the sequel, we will denote $X=P_{1}^{-1}, Y=P_{3}^{-1}$, and $Z=$ $-P_{3}^{-1} P_{2} P_{1}^{-1}$. We further restrict $M$ to the following case in order to obtain a linear matrix inequality (LMI) (see, e.g., [9]):

$$
M=\delta P^{T}\left[\begin{array}{c}
0 \\
B \mathcal{L} K
\end{array}\right],
$$

where $\delta$ is a scalar parameter. Pre- and postmultiply (10) by $\operatorname{diag}\left\{\left(P^{-1}\right)^{T}, P_{1}^{-1}, I\right\}$ and $\operatorname{diag}\left\{P^{-1}, P_{1}^{-1}, I\right\}$, respectively; also pre- and postmultiply (11) by $\operatorname{diag}\left\{\left(P^{-1}\right)^{T}, P_{1}^{-1}\right\}$ and $\operatorname{diag}\left\{P^{-1}, P_{1}^{-1}\right\}$ and denote

$$
\begin{aligned}
L=P_{1}^{-1} R P_{1}^{-1}, \quad F & =K P_{1}^{-1}, \quad \bar{S}=S^{-1}, \\
\left(P^{-1}\right)^{T} W P^{-1} & =\left[\begin{array}{cc}
\bar{W}_{1} & \bar{W}_{2} \\
* & \bar{W}_{3}
\end{array}\right] .
\end{aligned}
$$

Applying the Schur complement and expanding the block matrices, we obtain the following result under the assumptions made in Section 2. 
Theorem 2. Suppose that for a prescribed scalar $\delta$, there exist a scalar $\epsilon>0$, matrices $X>0, Y, Z, F, L>0, \bar{S}>$ $0, \bar{W}_{1}, \bar{W}_{2}, \bar{W}_{3}$, such that the following matrix inequalities are satisfied:

$$
\begin{gathered}
{\left[\begin{array}{cccc}
\Psi_{1} & \Psi_{2} & 0 & \Psi_{41} \\
* & \Psi_{3} & (1-\delta) B \mathcal{L} F & \Psi_{42} \\
* & * & -L & \Psi_{43} \\
* & * & * & \Psi_{5}
\end{array}\right]<0,} \\
{\left[\begin{array}{ccc}
\bar{W}_{1} & \bar{W}_{2} & 0 \\
* & \bar{W}_{3} & \delta B \mathcal{L} F \\
* & * & X \bar{S}^{-1} X
\end{array}\right] \geq 0,}
\end{gathered}
$$

where

$$
\begin{gathered}
\Psi_{1}=Z+Z^{T}+\mu L+\tau_{M} \bar{W}_{1}, \\
\Psi_{2}=Y+X(A-I)^{T}-Z^{T}+\tau_{M} \bar{W}_{2}+\delta(B \mathcal{L} F)^{T}, \\
\Psi_{3}=-Y-Y^{T}+\tau_{M} \bar{W}_{3}+\epsilon D D^{T}, \\
{\left[\begin{array}{c}
\Psi_{41} \\
\Psi_{42} \\
\Psi_{43}
\end{array}\right]=\left[\begin{array}{ccccc}
X E^{T} & \tau_{M} Z^{T} & 0 & X & Z^{T} \\
0 & \tau_{M} Y^{T} & 0 & 0 & Y^{T} \\
0 & 0 & F^{T} & 0 & 0
\end{array}\right],} \\
\Psi_{5}=\operatorname{diag}\left\{-\epsilon I,-\tau_{M} \bar{S},-Q_{2}^{-1},-Q_{1}^{-1},-X\right\} .
\end{gathered}
$$

Then, the control law

$$
u(k)=F X^{-1} x(k-\tau(k))
$$

is a guaranteed cost networked control law for system (1) and the corresponding cost function satisfies

$$
\begin{aligned}
J \leq & \lambda_{\max }\left(U^{T} X^{-1} U\right)+\rho_{1} \lambda_{\max }\left(U^{T} X^{-1} L X^{-1} U\right) \\
& +\rho_{2} \lambda_{\max }\left(U^{T} \bar{S}^{-1} U\right),
\end{aligned}
$$

where $\rho_{1}=\mu\left(\tau_{M}+\tau_{m}\right) / 2$ and $\rho_{2}=2 \tau_{M}\left(\tau_{M}+1\right)$.

Remark 4. From (22), we establish the following inequalities:

$$
\begin{gathered}
{\left[\begin{array}{cc}
-\alpha I & U^{T} \\
* & -X
\end{array}\right]<0, \quad\left[\begin{array}{cc}
-\beta I & U^{T} \\
* & -X L^{-1} X
\end{array}\right]<0,} \\
{\left[\begin{array}{cc}
-\gamma I & U^{T} \\
* & -\bar{S}
\end{array}\right]<0,}
\end{gathered}
$$

where $\alpha, \beta$, and $\gamma$ are scalars to be determined. It is worth noting that condition (23) is not an LMI because of the term $-X L^{-1} X$. This is also the case for condition (19) which is not an LMI because of the term $X \bar{S}^{-1} X$. Note that for any matrix $X>0$ we have

$$
X \bar{S}^{-1} X \geq 2 X-\bar{S}, \quad X L^{-1} X \geq 2 X-L
$$

Given a prescribed scalar $\delta$, the design problem of the optimal guaranteed cost controller can be formulated therefore as the following optimization problem:

$$
\begin{gathered}
\text { OP1: } \min _{\epsilon, X, Y, Z, F, L, \bar{S}_{1}, \bar{W}_{1}, \bar{W}_{2}, \bar{W}_{3}}\left(\alpha+\rho_{1} \beta+\rho_{2} \gamma\right) \\
\text { s.t. }\left\{\begin{array}{rrr}
\text { (i) } & \text { inequality }(18), \\
\text { (ii) } & {\left[\begin{array}{ccc}
\bar{W}_{1} & \bar{W}_{2} & 0 \\
* & \bar{W}_{3} & \delta B \mathcal{L} F \\
* & * & 2 X-\bar{S}
\end{array}\right] \geq 0,} \\
\text { (iii) } & {\left[\begin{array}{cc}
-\alpha I & U^{T} \\
* & -X
\end{array}\right]<0,\left[\begin{array}{cc}
-\beta I & U^{T} \\
* & -2 X+L
\end{array}\right]<0,} \\
& {\left[\begin{array}{ll}
-\gamma I & U^{T} \\
* & -\bar{S}
\end{array}\right]<0 .}
\end{array}\right.
\end{gathered}
$$

Clearly, the above optimization problem (25) is a convex optimization problem which can be effectively solved by existing LMI software [10]. Thus, the minimization of $\alpha+\rho_{1} \beta+\rho_{2} \gamma$ implies the minimization of the cost in (9). By applying a simple one-dimensional search over $\delta$ for a certain $\tau_{M}$, a global optimum cost can be found.

\subsection{Robust Stability with at least a fault-free actuator}

Based on the controller designed in Theorem 2, let us assume that actuator $i$ is fault-free, then we can redesign the $i$ th row of controller gain matrix $K$ to improve the robust performance for the system against actuator failures. We can rewrite the overall control system as

$$
x(k+1)=A_{1} x(k)+\left(B_{\bar{i}} \mathcal{L}_{\bar{i}} K_{\bar{i}}+b_{i} k_{i}\right) x(k-\tau(k)),
$$

where $A_{1}=A+D \Delta(k) E$, matrix $K_{\bar{i}}$ is obtained by deleting the $i$ th row from $K, B_{\bar{i}}$ is obtained by deleting the $i$ th column from $B$, and $\mathcal{L}_{\bar{i}}$ is obtained by deleting $i$ th row and $i$ th column from $\mathcal{L}$. The cost function associated to system (26) reads as

$$
J=\sum_{k=0}^{\infty} x_{e}^{T}(k) Q x_{e}(k)
$$

with $x_{e}^{T}(k)=\left[x^{T}(k), x^{T}(k-\tau(k))\right], Q=\operatorname{diag}\left\{Q_{1}, k_{i}^{T} Q_{2 i} k_{i}+\right.$ $\left.K_{\bar{i}}^{T} Q_{2 i} K_{\bar{i}}\right\}$, where $Q_{2 \bar{i}}$ is obtained by deleting the $i$ th row and $i$ th column from $Q_{2}$. With regard to system (26) where $K_{\bar{i}}$ is assumed to be known, we have the following result.

Theorem 3. If there exist a gain matrix $k_{i}$, a scalar $\epsilon>0$, symmetric positive-definite matrices $P_{1} \in \mathcal{R}^{n \times n}, R \in \mathcal{R}^{n \times n}, S \in$ $\mathcal{R}^{n \times n}$, and matrices $P_{2} \in \mathcal{R}^{n \times n}, P_{3} \in \mathcal{R}^{n \times n}, W \in \mathcal{R}^{2 n \times 2 n}$, 
$M \in \mathcal{R}^{2 n \times n}$ such that the following matrix inequalities are satisfied:

$$
\begin{gathered}
{\left[\begin{array}{ccc}
\Gamma & P^{T}\left[\begin{array}{c}
0 \\
B_{\bar{i}} \mathcal{L}_{\bar{i}} K_{\bar{i}}+b_{i} k_{i}
\end{array}\right]-M & {\left[\begin{array}{c}
E^{T} \\
0
\end{array}\right]} \\
* & -R+k_{i}^{T} Q_{2 i} k_{i}+K_{\bar{i}}^{T} Q_{2 i} K_{\bar{i}} & 0 \\
* & * & -\epsilon I
\end{array}\right]<0,} \\
{\left[\begin{array}{cc}
W & M \\
* & S
\end{array}\right] \geq 0,}
\end{gathered}
$$

then, system (26) is asymptotically stable and the cost function (27) satisfies inequality (13).

Proof. The proof is similar to the proof of Theorem 1.

\subsection{Step (ii): Controller Redesign}

Proceeding as in step (i), we restrict $M$ to the following case in order to obtain an LMI:

$$
M=\delta P^{T}\left[\begin{array}{c}
0 \\
b_{i} k_{i}
\end{array}\right]
$$

where $\delta$ is a scalar parameter. Pre- and postmultiply (28) with $\operatorname{diag}\left\{\left(P^{-1}\right)^{T}, P_{1}^{-1}, I\right\}$ and $\operatorname{diag}\left\{P^{-1}, P_{1}^{-1}, I\right\}$, respectively; also pre- and postmultiply (29) with $\operatorname{diag}\left\{\left(P^{-1}\right)^{T}, P_{1}^{-1}\right\}$ and $\operatorname{diag}\left\{P^{-1}, P_{1}^{-1}\right\}$, respectively, and denote

$$
\begin{aligned}
L=P_{1}^{-1} R P_{1}^{-1}, \quad F^{*} & =k_{i} P_{1}^{-1}, \quad \bar{S}=S^{-1}, \\
\left(P^{-1}\right)^{T} W P^{-1} & =\left[\begin{array}{cc}
\bar{W}_{1} & \bar{W}_{2} \\
* & \bar{W}_{3}
\end{array}\right] .
\end{aligned}
$$

The Schur complement trick leads to the following controller redesign result.

Theorem 4. Suppose that for a prescribed scalar $\delta$, there exist a scalar $\epsilon>0$, matrices $X>0, Y, Z, F^{*}, L>0, \bar{S}>0$, $\bar{W}_{1}, \bar{W}_{2}, \bar{W}_{3}$, such that the following matrix inequalities are satisfied:

$$
\begin{gathered}
{\left[\begin{array}{cccc}
\tilde{\Psi}_{1} & \tilde{\Psi}_{2} & 0 & \tilde{\Psi}_{41} \\
* & \widetilde{\Psi}_{3} & B_{i} \mathcal{L}_{i} K_{i} X+(1-\delta) b_{i} F^{*} & \widetilde{\Psi}_{42} \\
* & * & -L & \widetilde{\Psi}_{43} \\
* & * & * & \widetilde{\Psi}_{5}
\end{array}\right]<0,} \\
{\left[\begin{array}{ccc}
\bar{W}_{1} & \bar{W}_{2} & 0 \\
* & \bar{W}_{3} & \delta b_{i} F^{*} \\
* & * & X \bar{S}^{-1} X
\end{array}\right] \geq 0}
\end{gathered}
$$

where

$$
\begin{gathered}
\widetilde{\Psi}_{1}=Z+Z^{T}+\mu L+\tau_{M} \bar{W}_{1}, \\
\widetilde{\Psi}_{2}=Y+X(A-I)^{T}-Z^{T}+\tau_{M} \bar{W}_{2}+\delta\left(b_{i} F^{*}\right)^{T}, \\
\widetilde{\Psi}_{3}=-Y-Y^{T}+\tau_{M} \bar{W}_{3}+\epsilon D D^{T}, \\
{\left[\begin{array}{c}
\widetilde{\Psi}_{41} \\
\tilde{\Psi}_{42} \\
\widetilde{\Psi}_{43}
\end{array}\right]=\left[\begin{array}{cccccc}
X E^{T} & \tau_{M} Z^{T} & 0 & 0 & X & Z^{T} \\
0 & \tau_{M} Y^{T} & 0 & 0 & 0 & Y^{T} \\
0 & 0 & \left(F^{*}\right)^{T} & X K_{\bar{i}}^{T} & 0 & 0
\end{array}\right],} \\
\widetilde{\Psi}_{5}=\operatorname{diag}\left\{-\epsilon I,-\tau_{M} \bar{S},-Q_{2 i}^{-1},-Q_{2 i}^{-1},-Q_{1}^{-1},-X\right\} .
\end{gathered}
$$

Then, the ith control law

$$
u_{i}(k)=F^{*} X^{-1} x(k-\tau(k))
$$

is a guaranteed cost networked control law of system (26) and the corresponding cost function satisfies

$$
\begin{aligned}
J \leq & \lambda_{\max }\left(U^{T} X^{-1} U\right)+\rho_{1} \lambda_{\max }\left(U^{T} X^{-1} L X^{-1} U\right) \\
& +\rho_{2} \lambda_{\max }\left(U^{T} \bar{S}^{-1} U\right), \\
\text { where } \rho_{1} & =\mu\left(\tau_{M}+\tau_{m}\right) / 2 \text { and } \rho_{2}=2 \tau_{M}\left(\tau_{M}+1\right) .
\end{aligned}
$$

Given a prescribed scalar $\delta$, the redesign problem of the optimal guaranteed cost controller can be formulated as the following convex optimization problem:

$$
\begin{gathered}
\text { OP2: } \min _{\epsilon, X, Y, Z, F^{*}, L, \bar{S}_{1}, \bar{W}_{1}, \bar{W}_{2}, \bar{W}_{3}}\left(\alpha+\rho_{1} \beta+\rho_{2} \gamma\right) \\
\text { s.t. }\left\{\begin{aligned}
\text { inequality }(32), \\
\text { (ii) }\left[\begin{array}{ccc}
\bar{W}_{1} & \bar{W}_{2} & 0 \\
* & \bar{W}_{3} & \delta b_{i} F^{*} \\
* & * & 2 X-\bar{S}
\end{array}\right] \geq 0, \\
\text { (iii) }\left[\begin{array}{cc}
-\alpha I & U^{T} \\
* & -X
\end{array}\right]<0,\left[\begin{array}{cc}
-\beta I & U^{T} \\
* & -2 X+L
\end{array}\right]<0, \\
\\
{\left[\begin{array}{cc}
-\gamma I & U^{T} \\
* & -\bar{S}
\end{array}\right]<0 . }
\end{aligned}\right.
\end{gathered}
$$

\section{ILLUSTRATIVE EXAMPLE}

The dynamics are described by the following matrices:

$$
\begin{array}{ll}
A=\left[\begin{array}{cc}
0.9 & 0 \\
0.2 & 0.5
\end{array}\right], & B=\left[\begin{array}{cc}
0.2 & 0.1 \\
0 & -0.1
\end{array}\right], \\
D=\left[\begin{array}{cc}
0 & 0.1 \\
0.1 & 0
\end{array}\right], & E=\left[\begin{array}{cc}
0.1 & 0 \\
0.1 & -0.1
\end{array}\right],
\end{array}
$$

and the design parameters are chosen as

$$
Q_{1}=\left[\begin{array}{ll}
1 & 0 \\
0 & 1
\end{array}\right], \quad Q_{2}=\left[\begin{array}{cc}
0.1 & 0 \\
0 & 0.1
\end{array}\right], \quad U=\left[\begin{array}{ll}
1 & 0 \\
0 & 1
\end{array}\right] .
$$




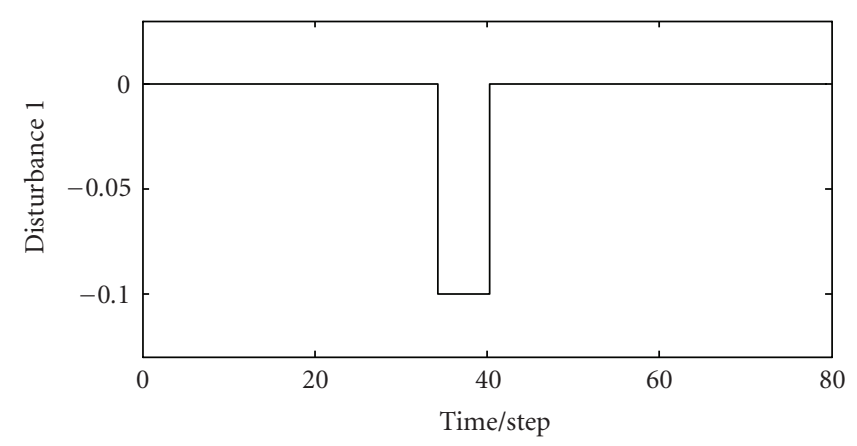

(a)

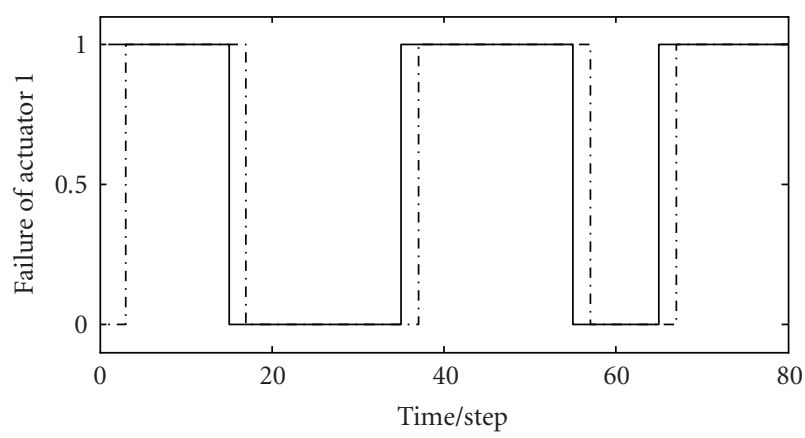

(c)

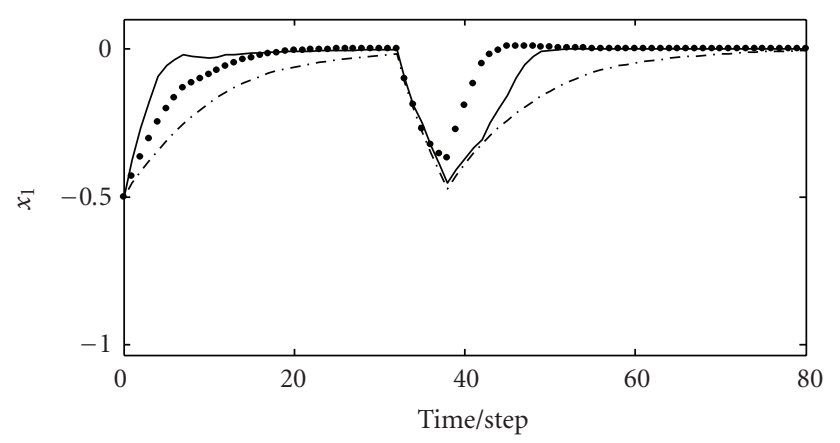

(e)

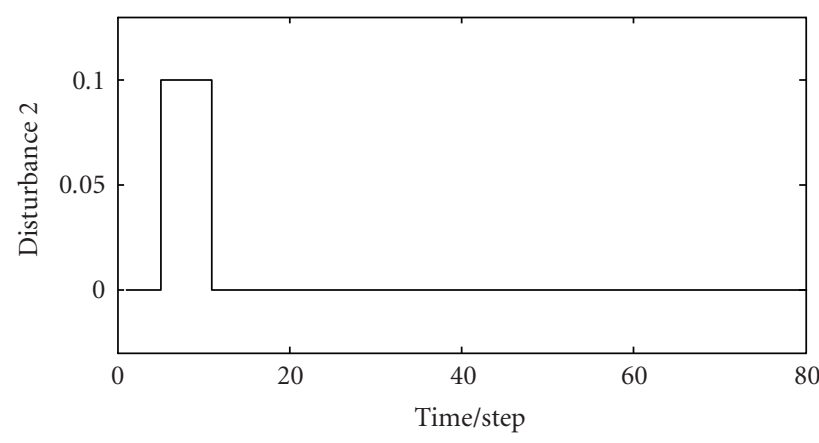

(b)

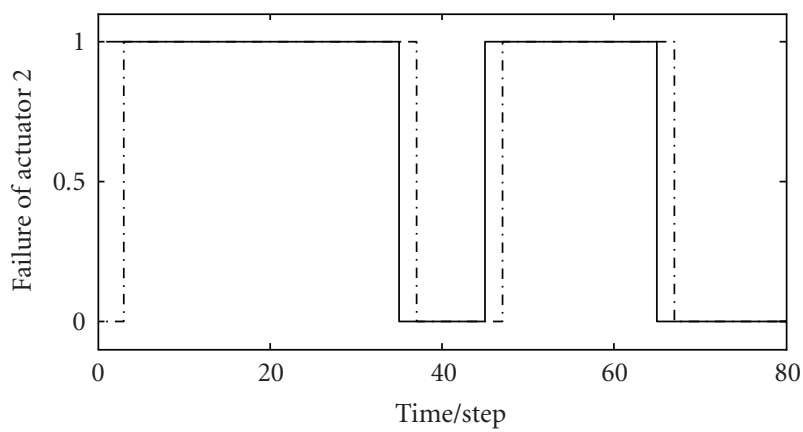

(d)

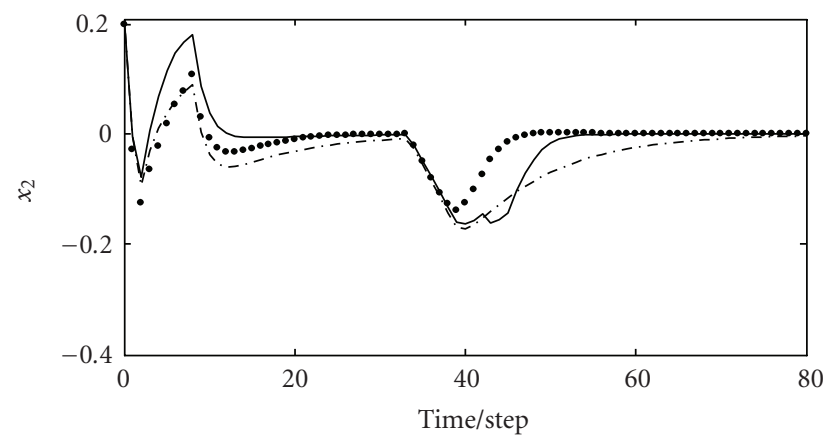

(f)

FIGURE 2: Disturbance, actuator failures, and state response.

When $\tau_{m}=1, \tau_{M}=2$, and $\delta=1$, by OP1 (25), the cost is obtained as $J_{1}=61.6653$ and the fault-tolerant controller can be designed for step (i):

$$
\left[\begin{array}{l}
k_{1} \\
k_{2}
\end{array}\right]=\left[\begin{array}{ll}
-0.0812 \times 10^{-5} & -0.1333 \times 10^{-5} \\
-0.1865 \times 10^{-5} & -0.3060 \times 10^{-5}
\end{array}\right] \text {. }
$$

In step (ii), by OP2 (37), the cost and the feedback gains are redesigned as

$$
\begin{array}{ll}
J_{2}=39.0026, & k_{1}^{*}=\left[\begin{array}{ll}
-0.8776 & -0.2857
\end{array}\right], \\
J_{3}=49.9616, & k_{2}^{*}=\left[\begin{array}{ll}
-0.6494 & -0.4161
\end{array}\right] .
\end{array}
$$

As a result, the two controllers are determined as follows:

$$
\begin{aligned}
& \# 1:\left[\begin{array}{l}
k_{1}^{*} \\
k_{2}
\end{array}\right]=\left[\begin{array}{cc}
-0.8776 & -0.2857 \\
-0.1865 \times 10^{-5} & -0.3060 \times 10^{-5}
\end{array}\right], \\
& \# 2:\left[\begin{array}{l}
k_{1} \\
k_{2}^{*}
\end{array}\right]=\left[\begin{array}{cc}
-0.0812 \times 10^{-5} & -0.1333 \times 10^{-5} \\
-0.6494 & -0.4161
\end{array}\right] .
\end{aligned}
$$

Figure 2 reports the simulation for two failures scenarios of the actuators. For this simulation, the time-varying normbounded uncertain matrix $\Delta(k)$ is taken as

$$
\Delta(k)=\left(\begin{array}{cc}
\sin (\pi k) & 0 \\
0 & \cos (\pi k)
\end{array}\right),
$$


which clearly satisfies the bound $\Delta(k)^{T} \Delta(k) \leq I$. The left column of Figure 2 is related to failures of actuator 1 and the right column is related to failures of actuator 2. Note also that for these two failures scenarios the system has been disturbed by step disturbances entering additively in the state equations. This is illustrated in the simulation where the step disturbance 1 shown in Figure 2(a) disturbs the state variables at time instant 35 and disappears at time instant 40 . The step disturbance 2 shown in Figure 2(b) disturbs the system at time instant 5 and disappears at time instant 10 . In Figure 2(c), the solid line represents the failure of actuator 1 which occurs at time instant 15 and disappears at time instant 35 , occurs again at time instant 55 and disappears at time instant 65 . In Figure 2(d), the solid line represents the failure of actuator 2 which occurs at time instant 35 and disappears at time instant 45 , occurs again at time instant 65 and disappears at time instant 80 . The delay of fault detection is assumed to be 3 time steps, which is represented by dot-dashed lines as shown in Figures 2(c) and 2(d). Under the above simulation setting, the state responses are shown in Figures 2(e) and 2(f):

(i) the dotted line represents the state response for controller-switching sequence $\mathrm{N}^{\circ} 1$ : \#2 is the initial controller, and \#1 is switched-on at time instant 38, then $\# 2$ at time instant $48, \# 1$ at time instant 68 ;

(ii) the solid line represents the state response for controller-switching sequence $\mathrm{N}^{\circ} 2: \# 1$ is the initial controller, and \#2 is switched-on at time instant 38, then \#1 at time instant $48, \# 2$ at time instant 68;

(iii) the dot-dashed line represents the state response under the fault tolerant control (i.e., robust control) of step (i).

The trace of matrices $\left(x^{*}\right)\left(x^{*}\right)^{T} /($ simulation time) is used as a performance measure for comparison, where $x^{*}$ represents the state trajectory in the different schemes and the simulation time is 80 seconds. After computation, we obtain for the above three control schemes the traces $T r_{1}=0.0279, T r_{2}=$ $0.0338, T r_{3}=0.0499$, which means that $T r_{1}<T r_{2}<T r_{3}$. We conclude that the proposed method for sequence $\mathrm{N}^{\circ} 1$ is the best control scheme. We also observe that for all possible switching sequences with controllers in the designed controllers bank, the proposed active FTC is able to guarantee at least the closed-loop stability of the overall system.

\section{CONCLUSION}

In this paper, the stability guaranteed active fault tolerant control against actuators failure in networked control system with time-varying but bounded delays has been addressed. Plants with norm-bounded parameter uncertainty have been considered, where the uncertainty may appear in the state matrix. Modelling network-induced delays as communication delays between sensors and actuators, linear memoryless state feedback fault-tolerant controllers have been developed through LMI-based methods. A simulation example has been presented to show the potentials of the proposed method for fault-tolerant control in networked control systems.

\section{APPENDIX}

\section{PROOF OF THEOREM 1}

The following matrix inequalities are essential for the proof of Theorem 1 .

Lemma 5 (see [11]). Assume that $a(\cdot) \in \mathcal{R}^{n_{a}}, b(\cdot) \in$ $\mathcal{R}^{n_{b}}$, and $N(\cdot) \in \mathcal{R}^{n_{a} \times n_{b}}$. Then, for any matrices $X \in$ $\mathcal{R}^{n_{a} \times n_{a}}, Y \in \mathcal{R}^{n_{a} \times n_{b}}$, and $Z \in \mathcal{R}^{n_{b} \times n_{b}}$, the following holds:

$$
-2 a^{T} N b \leq\left[\begin{array}{l}
a \\
b
\end{array}\right]^{T}\left[\begin{array}{cc}
X & Y-N \\
Y^{T}-N^{T} & Z
\end{array}\right]\left[\begin{array}{l}
a \\
b
\end{array}\right],
$$

where $\left[\begin{array}{cc}X & Y \\ Y^{T} & Z\end{array}\right] \geq 0$.

Lemma 6 (see [12]). Let $Y$ be a symmetric matrix and let $H, E$ be given matrices with appropriate dimensions, then

$$
Y+H F E+E^{T} F^{T} H^{T}<0
$$

holds for all $F$ satisfying $F^{T} F \leq I$, if and only if there exists a scalar $\epsilon>0$ such that

$$
Y+\epsilon H H^{T}+\epsilon^{-1} E^{T} E<0 .
$$

Proof. Note that $x(k-\tau(k))=x(k)-\sum_{l=k-\tau(k)}^{k-1} y(l)$, where $y(l)=x(l+1)-x(l)$. Then from system (8) we have

$$
0=\left(A_{1}+B \mathscr{L} K-I\right) x(k)-y(k)-B \mathscr{L} K \sum_{l=k-\tau(k)}^{k-1} y(l) .
$$

Choose the Lyapunov-Krasovskii function candidates as follows:

$$
V(k)=V_{1}(k)+V_{2}(k)+V_{3}(k),
$$

where

$$
\begin{aligned}
V_{1}(k)= & x^{T}(k) P_{1} x(k), \\
V_{2}(k)= & \sum_{l=k-\tau(k)}^{k-1} x^{T}(l) R x(l), \\
V_{3}(k)= & \sum_{\theta=-\tau_{M}}^{-1} \sum_{l=k+\theta}^{k-1} y^{T}(l) S y(l) \\
& +\sum_{\theta=-\tau_{M}+2}^{-\tau_{m}+1} \sum_{l=k+\theta-1}^{k-1} x^{T}(l) R x(l)
\end{aligned}
$$

Taking the forward difference for the Lyapunov functional $V_{1}(k)$, we have

$$
\Delta V_{1}(k)=2 x^{T}(k) P_{1} y(k)+y^{T}(k) P_{1} y(k) .
$$

From (A.4), we have

$$
2 x^{T}(k) P_{1} y(k)=2 \eta^{T}(k) P^{T}\left[\begin{array}{c}
y(k) \\
N-B \mathscr{L} K \sum_{l=k-\tau(k)}^{k-1} y(l)
\end{array}\right],
$$


where $N=\left(A_{1}+B \mathcal{L} K-I\right) x(k)-y(k), \eta^{T}(k)=\left[x^{T}(k) y^{T}(k)\right]$. Choose constant matrices $W, M$, and $S$ satisfying (11); by Lemma 5, we have

$$
\begin{aligned}
& -2 \sum_{l=k-\tau(k)}^{k-1} \eta^{T}(k) P^{T}\left[\begin{array}{c}
0 \\
B \mathcal{L} K
\end{array}\right] y(l) \\
& \leq \tau_{M} \eta^{T}(k) W \eta(k)+\sum_{l=k-\tau_{M}}^{k-1} y^{T}(l) S y(l) \\
& \quad+2 \eta^{T}(k)\left\{M-P^{T}\left[\begin{array}{c}
0 \\
B \mathcal{L} K
\end{array}\right]\right\}(x(k)-x(k-\tau(k)) .
\end{aligned}
$$

Similarly,

$$
\begin{aligned}
\Delta V_{2}(k)= & x^{T}(k) R x(k)-x^{T}(k-\tau(k)) R x(k-\tau(k)) \\
& +\sum_{l=k+1-\tau(k+1)}^{k-1} x^{T}(l) R x(l)-\sum_{k-\tau(k)+1}^{k-1} x^{T}(l) R x(l) .
\end{aligned}
$$

Note that

$$
\begin{aligned}
& \sum_{l=k+1-\tau(k+1)}^{k-\tau_{m}} x^{T}(l) R x(l) \\
& \quad=\sum_{l=k+1-\tau_{m}}^{k-1} x^{T}(l) R x(l)+\sum_{l=k+1-\tau(k+1)}^{k-\tau_{m}} x^{T}(l) R x(l) \\
& \quad \leq \sum_{l=k+1-\tau(k)}^{k-1} x^{T}(l) R x(l)+\sum_{l=k+1-\tau_{M}}^{k-\tau_{m}} x^{T}(l) R x(l) .
\end{aligned}
$$

So, we have

$$
\begin{aligned}
\Delta V_{2}(k) \leq & x^{T}(k) R x(k)-x^{T}(k-\tau(k)) R x(k-\tau(k)) \\
& +\sum_{l=k+1-\tau_{m}}^{k-\tau_{m}} x^{T}(l) R x(l) .
\end{aligned}
$$

Furthermore, we have

$$
\begin{aligned}
\Delta V_{3}(k)= & \tau_{M} y^{T}(k) S y(k)-\sum_{l=k+1-\tau_{m}}^{k-1} y^{T}(l) S y(l) \\
& +\left(\tau_{M}-\tau_{m}\right) x^{T}(k) R x(k)-\sum_{l=k+1-\tau_{m}}^{k-\tau_{m}} x^{T}(l) R x(l) .
\end{aligned}
$$

Combining (9) and (A.7)-(A.13), we have

$$
\begin{aligned}
\Delta V(k) \leq & \xi^{T}(k)\left[\Theta_{0}\left(\tau_{m}, \tau_{M}\right)+\hat{D} \Delta(k) \hat{E}+\widehat{E}^{T} \Delta^{T}(k) \hat{D}^{T}\right] \xi(k) \\
& -x_{e}^{T}(k) Q x_{e}(k),
\end{aligned}
$$

where

$$
\begin{aligned}
& \xi^{T}(k)=\left[\begin{array}{ll}
\eta^{T}(k) & x^{T}(k-\tau(k))
\end{array}\right], \\
& \widehat{D}^{T}=\left[\left[0 D^{T}\right] P \quad 0\right], \widehat{E}=[[E 0] 0], \\
& \Theta_{0}\left(\tau_{m}, \tau_{M}\right)=\left[\begin{array}{cc}
\Gamma_{0} & P^{T}\left[\begin{array}{c}
0 \\
B \mathcal{L} K
\end{array}\right]-M \\
* & -R+K^{T} Q_{2} K
\end{array}\right] \text {, } \\
& \Gamma_{0}=\Gamma-\epsilon P^{T}\left[\begin{array}{cc}
0 & 0 \\
0 & D D^{T}
\end{array}\right] P .
\end{aligned}
$$

By Lemma 6, we have

$$
\begin{aligned}
\Delta V(k) \leq & \xi^{T}(k)\left[\Theta_{0}\left(\tau_{m}, \tau_{M}\right)+\epsilon \hat{D} \hat{D}^{T}+\epsilon^{-1} \widehat{E}^{T} \hat{E}\right] \xi(k) \\
& -x_{e}^{T}(k) Q x_{e}(k) .
\end{aligned}
$$

It is worth observing that matrix $\left[\Theta_{0}\left(\tau_{m}, \tau_{M}\right)+\epsilon \hat{D} \hat{D}^{T}+\right.$ $\left.\epsilon^{-1} \widehat{E}^{T} \hat{E}\right]$ is the Schur complement of $-\epsilon I$ in the matrix of the left-hand side of inequality (10). Therefore, the negative definiteness of matrix $\left[\Theta_{0}\left(\tau_{m}, \tau_{M}\right)+\epsilon \hat{D} \hat{D}^{T}+\epsilon^{-1} \widehat{E}^{T} \hat{E}\right]$ resulting from inequality (10) implies that

$$
\Delta V(k) \leq-x_{e}^{T}(k) Q x_{e}(k)
$$

Summing both sides of the above inequality from 0 to $\infty$ leads to

$$
\begin{aligned}
\sum_{k=0}^{\infty} \Delta V(k) & =V(\infty)-V(0) \\
& \leq-\sum_{k=0}^{\infty} x_{e}^{T}(k) Q x_{e}(k)=-J
\end{aligned}
$$

which, from system stability, yields

$$
J \leq V(0)
$$

that is, inequality (13).

\section{ACKNOWLEDGMENTS}

The authors would like to thank the anonymous reviewers for their constructive comments and suggestions that have improved the quality of the manuscript. This work is supported by the French National Research Agency (Agence Nationale de la Recherche, ANR) project SafeNECS (SafeNetworked Control Systems) under Grant no. ANR-ARA SSIA-NV-15.

\section{REFERENCES}

[1] M. Blanke, M. Kinnaert, J. Lunze, and M. Staroswiecki, Diagnosis and Fault-Tolerant Control, Springer, Berlin, Germany, 2003.

[2] M. Mariton, "Detection delays, false alarm rates and the reconfiguration of control systems," International Journal of Control, vol. 49, no. 3, pp. 981-992, 1989. 
[3] M. Mahmoud, J. Jiang, and Y. Zhang, "Stabilization of active fault tolerant control systems with imperfect fault detection and diagnosis," Stochastic Analysis and Applications, vol. 21, no. 3, pp. 673-701, 2003.

[4] N. E. Wu, "Robust feedback design with optimized diagnostic performance," IEEE Transactions on Automatic Control, vol. 42, no. 9, pp. 1264-1268, 1997.

[5] M. Maki, J. Jiang, and K. Hagino, "A stability guaranteed active fault-tolerant control system against actuator failures," International Journal of Robust and Nonlinear Control, vol. 14, no. 12, pp. 1061-1077, 2004.

[6] W. Zhang, M. S. Branicky, and S. M. Phillips, "Stability of networked control systems," IEEE Control Systems Magazine, vol. 21, no. 1, pp. 84-99, 2001.

[7] P. Antsaklis and J. Baillieul, "Special issue on technology of networked control systems," Proceedings of the IEEE, vol. 95, no. 1, pp. 5-8, 2007.

[8] Y. Tipsuwan and M.-Y. Chow, "Control methodologies in networked control systems," Control Engineering Practice, vol. 11, no. 10, pp. 1099-1111, 2003.

[9] W.-H. Chen, Z.-H. Guan, and X. Lu, "Delay-dependent guaranteed cost control for uncertain discrete-time systems with delay," IEE Proceedings: Control Theory and Applications, vol. 150, no. 4, pp. 412-416, 2003.

[10] P. Gahinet, A. Nemirovsky, A. J. Laub, and M. Chilali, LMI Control Toolbox - for Use with Matlab, The Math Works, Natick, Mass, USA, 1995.

[11] Y. S. Moon, P. Park, W. H. Kwon, and Y. S. Lee, "Delaydependent robust stabilization of uncertain state-delayed systems," International Journal of Control, vol. 74, no. 14, pp. 1447-1455, 2001.

[12] Y. Wang, L. Xie, and C. E. de Souza, "Robust control of class uncertain nonlinear systems," Systems and Control Letters, vol. 19, no. 2, pp. 139-149, 1992. 

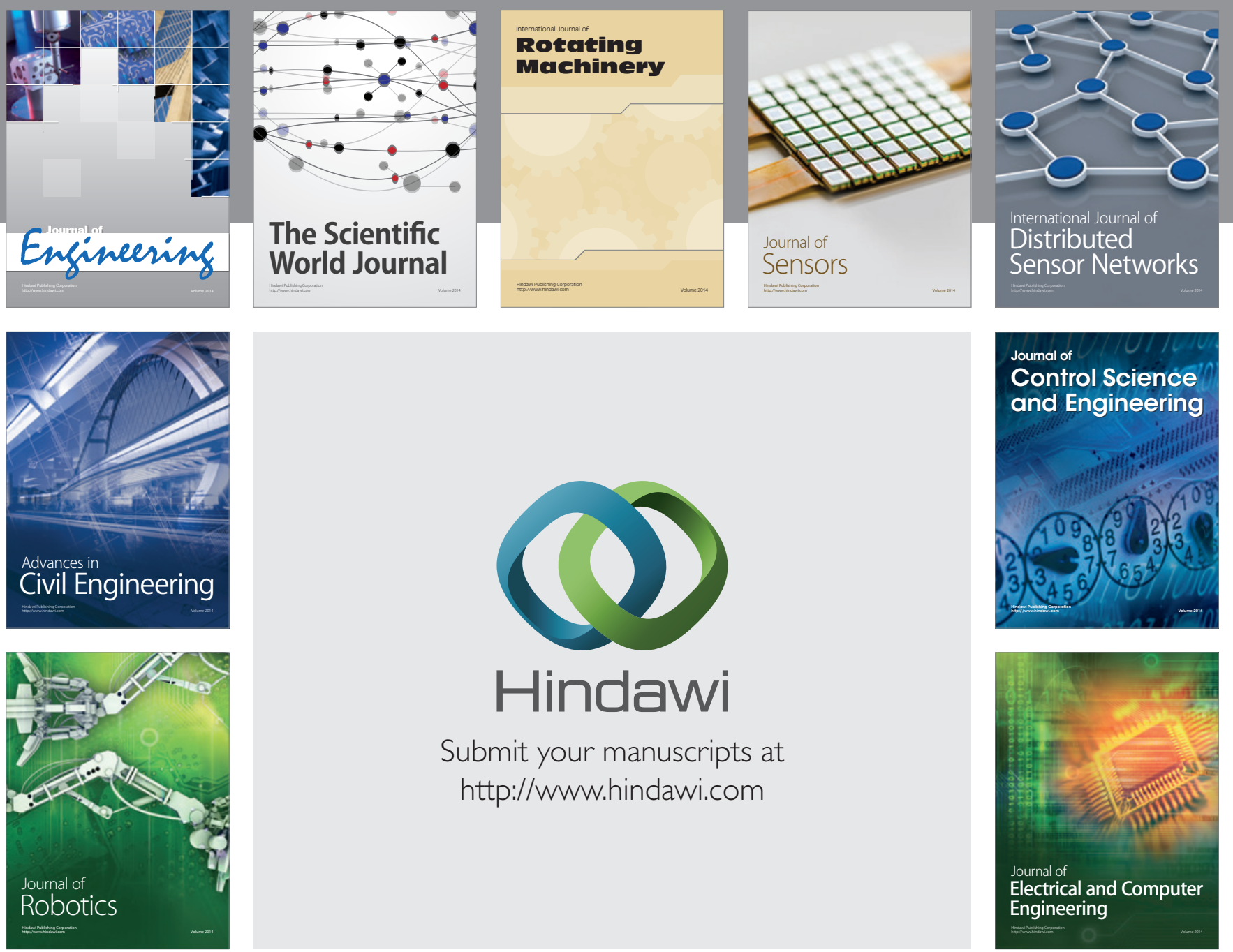

Submit your manuscripts at

http://www.hindawi.com
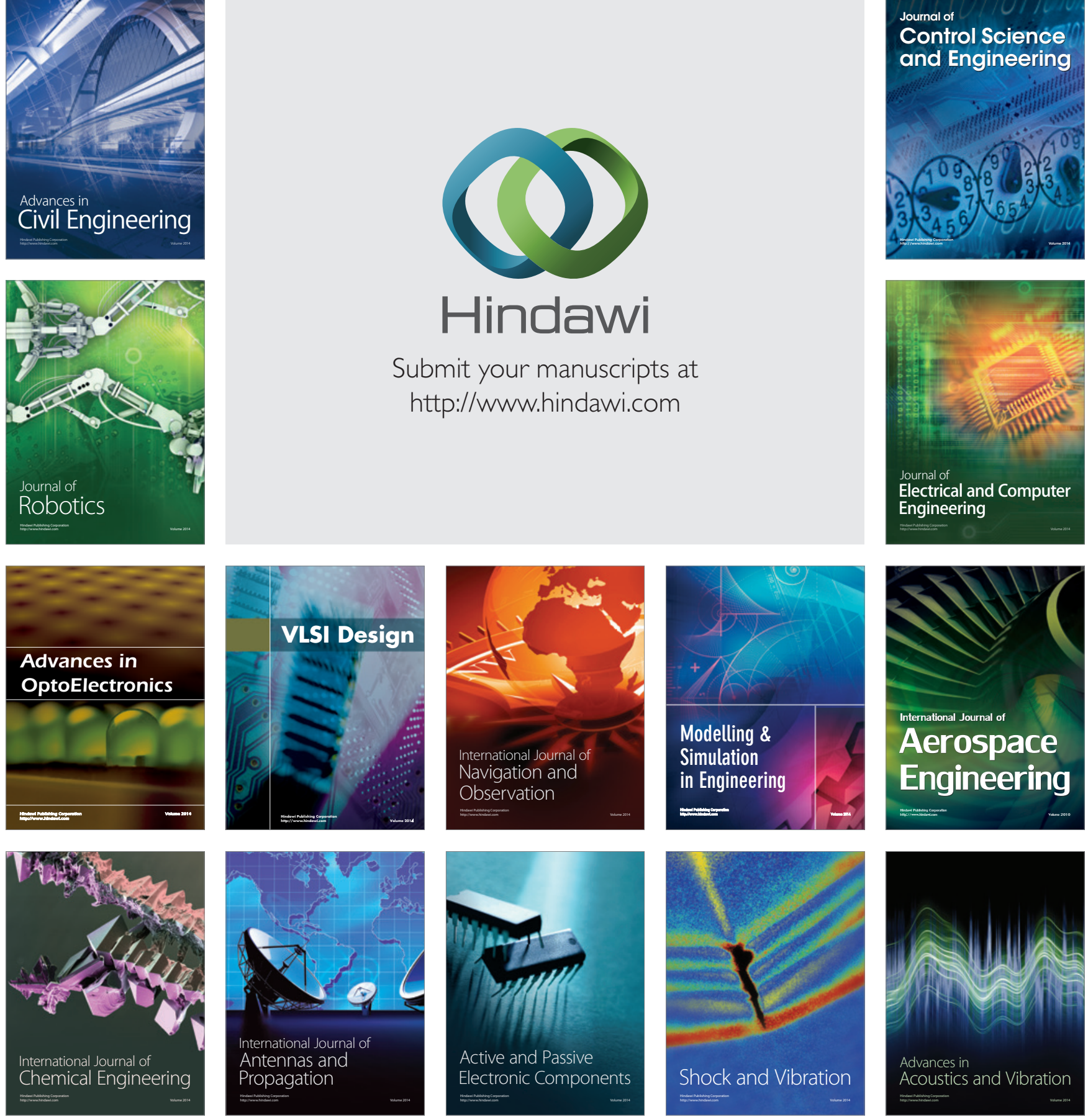